\title{
La economía del deporte en España: Análisis bibliométrico de una década (2002-2011)
}

\author{
JOSÉ MANUEL SÁNCHEZ SANTOS \\ Departamento de Economía Aplicada I, UNIVERSIDAD DE A CORUÑA, ESPAÑA. E- \\ mail: jose.manuel.sanchez@udc.es
}

PABLO CASTELLANOS GARCÍA

Departamento de Economía Aplicada I, UNIVERSIDAD DE A CORUÑA, ESPAÑA. Email:pcg@udc.es

\begin{abstract}
RESUMEN
En este artículo se presenta un estudio descriptivo, basado en datos bibliométricos, que permite valorar la evolución de la investigación en economía del deporte en España durante el período 2002-2011. A lo largo de esta década, la investigación en economía del deporte desarrollada por investigadores españoles ha registrado avances significativos. Esto ha sido posible gracias a la constitución de una importante comunidad de profesores investigadores en algunas universidades españolas. Nuestra principal conclusión es que, a pesar de que en nuestro país la economía del deporte todavía está en fase emergente y se concentra en un número relativamente reducido de autores e instituciones, ha mejorado notablemente en los últimos años, tanto en términos de cantidad como de calidad. Como consecuencia de los mencionados avances la investigación en este campo ha logrado posicionarse como una de las disciplinas en la que España tiene peso específico a nivel internacional.
\end{abstract}

Palabras clave: Análisis bibliométrico, economía del deporte.

\section{Sports Economics in Spain: A Bibliometric Analysis for a Decade (2002-2011)}

\begin{abstract}
In this paper we show a descriptive study, based on bibliometric data that allow us to value the evolution of the research in sports economics in Spain during the period 2002-2011. Throughout this decade, research in sports economics developed by Spanish investigators has reached significant advances. This has been possible due to the constitution of an important community of investigators in some Spanish universities. Our main conclusion is that, although in our country sports economics is still in an emergent phase and it is concentrated on a relatively reduced number of authors and institutions, it has improved notably in the last years, both in terms of quantity and quality. As a consequence of the aforementioned advances, the research in this field has been able to be positioned as one of the disciplines in which Spain has specific weight at international level.
\end{abstract}

Keywords: Bibliometric Analysis, Sports Economics.

Clasificación JEL: L83

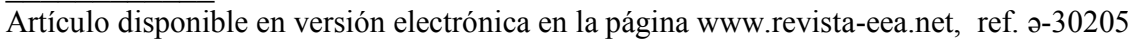




\section{INTRODUCCIÓN}

La aproximación al deporte con las herramientas de análisis propias de la Economía ha dado lugar a un campo de investigación específico, la economía del deporte, que está ampliando su presencia y reconocimiento en la comunidad académica internacional. Jewell (2006) pone de manifiesto cómo la economía del deporte ha pasado de ser un ejercicio ocasional de divertimento a ocupar un lugar destacado y legitimado dentro de la Economía como disciplina. Como prueba de ello, este mismo autor examina a una serie de artículos publicados en las revistas de economía de mayor prestigio, destacando lo innovador de las metodologías empleadas en los mismos así como el rigor de la argumentación que los caracteriza.

Cuestiones tales como los determinantes económicos del éxito deportivo, el impacto económico de los eventos deportivos, el análisis económico de los deportes profesionales de equipo, el mercado de trabajo de los deportistas profesionales, la demanda de deporte, el balance competitivo, etc. son temas que han ido adquiriendo un protagonismo creciente en las agendas investigadoras de un numeroso grupo de economistas.

Si bien existe un consenso generalizado en considerar como punto de partida de esta disciplina el artículo de Rottenberg (1956), no obstante, no es hasta la década de los 80 del siglo pasado cuando la literatura académica sobre economía del deporte despega definitivamente. Así pues, estamos hablando de una disciplina relativamente reciente. En este sentido, probablemente el indicador más significativo del auge y reconocimiento de la misma es la aparición del Journal of Sports Economics en el año 2000.

Recientemente Sánchez y Castellanos (2011) han abordado la medición y el análisis de la producción científica en economía del deporte a nivel internacional, estudiando diversos aspectos de aquella. Entre otros resultados, el análisis bibliométrico llevado a cabo por estos autores constata que en los últimos años han crecido exponencialmente los artículos sobre temas relacionados con el deporte publicados en revistas académicas de impacto. Paralelamente, también ha aumentado la oferta académica de cursos de economía del deporte, se han publicado un buen número de manuales e igualmente es significativo el incremento de las sesiones en congresos de economía que se centran en el análisis económico del deporte (Fizel, 2006).

Las anteriores consideraciones son aplicables al ámbito internacional, en el que parece evidente que los investigadores en economía sacan provecho de las oportunidades únicas que ofrece la industria del deporte tanto para el análisis teórico como empírico. Ésta es precisamente una de las razones que suele esgrimirse para explicar el crecimiento de este campo de investigación. Una vez constatada la consolidación de la economía del deporte a nivel internacional, 
cabe plantearse en qué medida la comunidad académica española es partícipe de esta tendencia y no se ha mantenido al margen de esta evolución.

Si más arriba apuntábamos que la economía del deporte es una disciplina con una corta historia, en el caso de nuestro país lo es todavía más. Un dato respalda esta afirmación: han transcurrido apenas diez años desde que García y Rodríguez (2002) publicaron en el Journal of Sport Economics el que podríamos catalogar como primer artículo de investigadores españoles en una revista internacional de impacto. ${ }^{1}$ Una década es un período de tiempo corto, pero suficiente para poner de manifiesto que la economía del deporte en España es una disciplina emergente y que se encuentra en plena fase de consolidación.

Precisamente, con el fin de ofrecer una visión del estado en el que se encuentra la investigación sobre economía del deporte en España, en este artículo se aborda el estudio de la evolución y el estado actual de la producción científica en economía del deporte en España, entendiendo por tal la realizada por investigadores españoles y publicada en revistas científicas indexadas. En última instancia, el objetivo es identificar los autores y las universidades más implicadas en la investigación y en la publicación en este campo, el posicionamiento internacional, el impacto de dicha investigación, las temáticas más abordadas, las metodologías de análisis empleadas, así como las futuras oportunidades de investigación.

Para llevar a cabo esta evaluación preliminar, seguimos las directrices de elaboración de un estudio bibliométrico estándar, de tal modo que nuestro trabajo se estructura del siguiente modo. En la segunda sección se realizan algunas consideraciones relativas a la delimitación analítica de la disciplina. En la tercera, se detalla el proceso de selección del objeto de análisis (el conjunto de revistas que serán base de nuestro estudio), se acota temporalmente la investigación y se especifican las unidades de medida (autores, instituciones, artículos y páginas). En la cuarta sección se resumen e interpretan los principales resultados del análisis bibliométrico. Por último, a modo de síntesis se ofrecen una serie de consideraciones finales.

\section{DELIMITACIÓN ANALÍTICA DE LA DISCIPLINA}

El primer paso de este análisis bibliométrico consiste en la acotación analítica de la economía del deporte. Para proceder a la delimitación de las fronteras analíticas de esta disciplina, nos hemos basado en las opiniones de algunos de los expertos que gozan de mayor reconocimiento en este campo.

\footnotetext{
${ }^{1}$ La búsqueda de los antecedentes más lejanos en el tiempo nos remite a dos comunicaciones presentadas en la VII Reunión de Asepelt-España por Sergio Quessada y M. Dolores Díez, tituladas, respectivamente: Economía Pública del deporte no profesional y Economía del deporte: un nuevo sector en la investigación social europea.
} 
En este sentido, dentro del ámbito de la investigación relacionada con el deporte, Jewell (2006) distingue dos grandes líneas no excluyentes: sports microeconomics y sportometrics. Según este autor, por un lado, estaría la investigación que aplica la teoría microeconómica a la industria del deporte en el intento de entender el funcionamiento de los mercados deportivos. A esta línea la denomina "microeconomía del deporte" y en ella se incardinarían los trabajos que desarrollan o adaptan la teoría económica con el objetivo de entender el funcionamiento de la industria del deporte. Por otro lado, existe una línea de investigación que se caracteriza fundamentalmente por usar los datos que ofrece el mundo del deporte para verificar o explicar varias hipótesis de la teoría microeconómica convencional. Fueron Goff y Tollison (1990) quienes acuñaron para esta segunda línea el término de sportometrics, lo que sería en cierta medida equiparable a una economía experimental que se basa en los datos de las competiciones deportivas de manera similar a como se utilizan los datos derivados de la realización de experimentos en laboratorios. Más en concreto, estos autores emplean el término sportometrics para referirse a la aplicación de la teoría económica al comportamiento de los deportistas para explicar cómo actúan y para ver si lo que hacen puede ayudar a entender la conducta de los agentes en otros entornos profesionales. Desde este punto de vista, el deporte es un campo útil para el desarrollo de la teoría económica en la medida en que constituye un entorno en el que los agentes implicados (fundamentalmente, pero no sólo, deportistas) responden a incentivos y están sujetos a restricciones claramente definidas.

Para Fort (2008), el rasgo distintivo de la economía del deporte es el uso del análisis económico para estudiar el deporte. Por su parte, Coates (2008) considera que la economía del deporte se define por la aplicación de la teoría de la decisión o de los precios y, de acuerdo con su planteamiento, cualquier trabajo que aborde el deporte usando funciones de incentivos y funciones objetivo o que intente comprender, explicar o predecir las elecciones de los agentes en un contexto deportivo, puede considerarse como economía del deporte. En una línea similar, Berri y Schmidt (2010) plantean que se puede aprender sobre la racionalidad o irracionalidad de la toma de decisiones estudiando el mundo del deporte. De hecho, Berri (2007) sostiene que la investigación sobre cómo se procesa la información por los responsables de la toma de decisiones es el área más importante en la que la economía del deporte puede realizar aportaciones más relevantes en el ámbito de la Economía. Zimbalist (2001) destaca que la esencia de la economía del deporte es la aplicación de las herramientas analíticas de la organización industrial, economía laboral, finanzas públicas y elección pública, teoría micro y macroeconómica, teoría de los juegos, teoría de las subastas y econometría, entre otras.

En suma, esta diversidad de perspectivas lo que viene a poner de manifiesto es que las fronteras de la disciplina no son del todo nítidas ni precisas. Así pues, 
el proceso de delimitación analítica inevitablemente conlleva la utilización de criterios arbitrarios. Por ejemplo, es difícil trazar fronteras que separen economía del deporte, gestión deportiva y/o estadística del deporte. No obstante, en nuestro caso y como criterio para seleccionar los trabajos que pueden ser considerados como economía del deporte, partimos de una definición amplia y operativa, en la cual se incluye cualquier trabajo que responda a alguna de las anteriores definiciones. Específicamente, a efectos del presente análisis bibliométrico, economía del deporte es cualquier estudio que emplee metodología económica y/o econométrica con el fin de analizar: (i) los datos del mundo del deporte, (ii) un mercado deportivo o relacionado con el deporte (mercado de trabajo, derechos de retransmisión, apuestas deportivas, etc.) (iii) y/o el comportamiento de los agentes implicados (deportistas, entrenadores, aficionados, árbitros, administraciones públicas, etc.).

\section{SELECCIÓN DE LA MUESTRA OBJETO DE ANÁLISIS Y ACOTACIÓN TEMPORAL DE LA INVESTIGACIÓN}

Como objeto de estudio del análisis bibliométrico que llevamos a cabo, hemos tomado las publicaciones en revistas de carácter científico, entendiendo por tales aquellas publicaciones que cumplen unos criterios mínimos de calidad, en concreto, el de estar indexadas en determinadas bases de datos:

a) En primer lugar se ha tomado la muestra de revistas de la Social Science Citation Index Database (SSCI), considerada una de las bases bibliográficas más prestigiosas en Ciencias Sociales, debido a los elevados estándares exigidos para su inclusión y también por el cálculo del índice de impacto científico de las revistas, que aparece publicado en el Journal Citation Reports (JCR).

b) En segundo lugar se ha utilizado la base de datos EconLit para seleccionar todas las publicaciones sobre deporte en las que participan investigadores españoles. Consideramos que por tratarse de una base de datos de revistas de economía, las publicaciones sobre deporte encajarían en la delimitación amplia de la disciplina establecida en la sección anterior.

c) En tercer lugar se ha realizado la búsqueda en Scopus, una base de datos bibliográfica de resúmenes y citas de artículos de revistas científicas, editada por Elsevier.

d) Por último, hemos completado nuestro análisis realizando una revisión sistemática de revistas especializadas que no están incluidas en las anteriores bases de datos, pero que cuentan con un sistema de revisión por pares acreditado. En concreto, hemos extendido la búsqueda al International Journal of Sport Management and Marketing, Journal of Quantitative Analysis in Sports e International Journal of Sports Marketing \& Sponsorship. 
Como aclaración adicional relativa al desarrollo del proceso de búsqueda, cabe señalar que inicialmente la realizamos en la base de datos SSCI rastreando en las categorías de Economics, Business y Management and Finance. No obstante, en la medida en que es bastante habitual que artículos cuyo contenido es susceptible de ser catalogado como economía del deporte se publiquen en revistas incluidas en otras categorías, extendimos nuestro análisis a otras áreas como Urban Studies, Public Administration o Sports Sciences. Además, dado que también se publican artículos en revistas que no están clasificadas en el campo de las Ciencias Sociales, ampliamos la búsqueda a las revistas incluidas en el Science Citation Index (SCI). Por su parte, en Scopus, nos centramos en las categorías Economics, Econometrics and Finance y Business, Management and Accounting.

Con esta elección del cuerpo de revistas en el que centramos el proceso de búsqueda pretendemos generar una muestra representativa de la producción científica en economía del deporte en la que participan autores españoles. Somos conscientes de que la decisión de incluir/excluir algunas revistas y artículos basándonos en el criterio adoptado tiene un componente de arbitrariedad, pero entendemos que no compromete el objetivo de identificar los autores más prolíficos en este campo de estudio ${ }^{2}$.

La búsqueda se llevó a cabo en dos fases. La primera consistió en un análisis sistemático de las principales bases de datos mencionadas anteriormente, utilizando descriptores o palabras clave. Esta búsqueda inicial produjo una lista de autores españoles que contaban con alguna publicación sobre la materia. En una segunda fase, una vez identificados los autores, procedimos a la revisión de todas las publicaciones de los mismos para confirmar que todas las que cumplían los criterios de selección estaban siendo consideradas.

Como consecuencia de este proceso de búsqueda, configuramos una base de datos compuesta inicialmente por 73 trabajos (70 artículos más 3 capítulos de libros) cuya publicación se extiende a largo del período 2002-2011.

Por lo que se refiere a los autores, se ha decidido considerar la nacionalidad española de los mismos y, por tanto, se analiza la producción científica de investigadores españoles, con residencia en España o en el extranjero. En cuanto a la institución a la que se asignan los autores, es la primera que aparece o la más frecuente, si son varias las publicaciones.

\footnotetext{
${ }^{2}$ A título de ejemplo, nos encontramos con trabajos de algunos de los autores de la muestra publicados en los monográficos que en su día editaron Revista Econòmica de Catalunya y Revista Asturiana de Economía, o en otras revistas como Revista de Empresa, Capital Humano, Revista Jurídica de Deporte y Entretenimiento, Decisión, etc. Estas referencias no han sido computadas, al no estar las mencionadas revistas indexadas en las bases de datos bibliográficas tomadas como referencia en el momento de la realización de nuestro estudio.
} 
En lo que respecta a las coautorías, se ha optado por construir dos indicadores de autores y páginas ponderadas, donde se prorratea el valor absoluto de cada variable original entre el número de participantes en la coautoría.

\section{RESULTADOS}

Basándonos en los criterios que acabamos de exponer, procedimos a realizar el estudio bibliométrico de la producción científica en economía del deporte realizada por investigadores españoles entre 2002 y 2011.

\subsection{Producción científica}

Tal y como hemos señalado anteriormente, el proceso de búsqueda arrojó un total de 73 trabajos publicados en 33 revistas. De este total de trabajos, 70 son artículos publicados en revistas y 3 son capítulos de libros que aparecen indexados en EconLit. En conjunto, en los 73 trabajos incluidos en este estudio han participado 83 autores españoles y se han generado un total de 1.145 páginas. Por su parte, la distribución de documentos por idioma de publicación revela que el principal es el inglés, si bien este dato no es muy significativo considerando el lugar de publicación de la mayoría de las revistas indexadas en esta base.

Para el cómputo de la actividad científica se ha utilizado el número de artículos (Gráfico 1) y el de páginas (Gráfico 2). La evolución de ambas variables que se refleja en estos gráficos permite constatar que la investigación en economía del deporte aflora a principios de los años 2000 e inicia su despegue a mediados de esta década. Por lo tanto, aunque en términos absolutos la presencia de la investigación en economía del deporte puede parecer todavía escasa, es preciso tener en cuenta que nos encontramos ante un intervalo temporal corto y en el que, además, el 72,6\% de los trabajos se publica en los últimos cinco años de la muestra. En particular, se observa un salto cualitativo en 2006, año a partir del cual crece la investigación sin apenas altibajos, registrando los mayores volúmenes de producción en los últimos dos años de la muestra (2010 y 2011). De hecho, en el último año prácticamente se duplica la producción del año anterior. Estos datos preliminares de carácter descriptivo apuntan a una disciplina que se podría calificar de emergente y que se encuentra en plena fase de consolidación. 


\section{Gráfico 1}

Evolución de la producción científica española en economía del deporte: $n^{\circ}$ de artículos

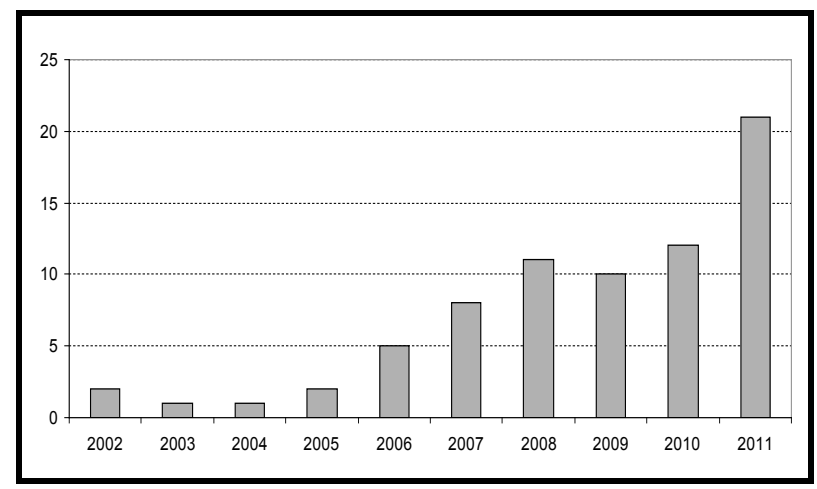

Fuente: Elaboración propia.

Gráfico 2

Evolución de la producción científica española en economía del deporte: volumen de páginas

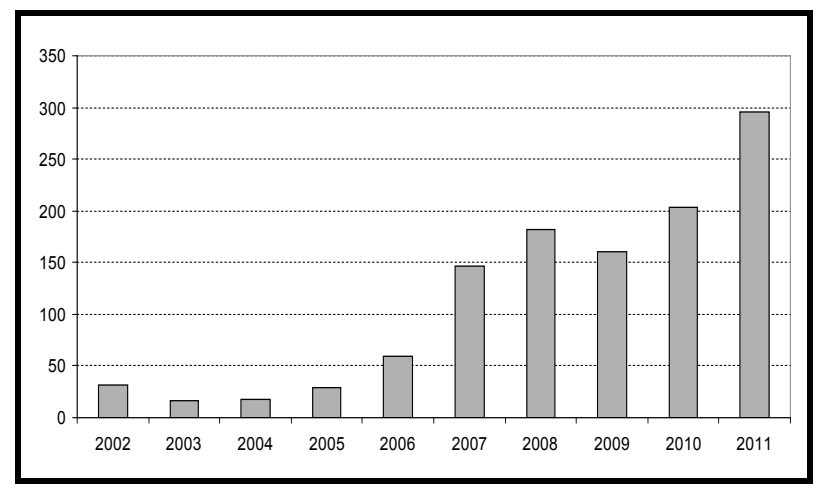

Fuente: Elaboración propia.

\subsection{Posición internacional}

Para la valoración de la presencia internacional de la producción científica española en economía del deporte, utilizamos los datos del SCI que sirvieron de base para el análisis bibliométrico de Sánchez y Castellanos (2011). La distribución geográfica de la producción científica en esta disciplina coloca a España en una posición relevante a nivel internacional y, especialmente, en el ámbito europeo (Sánchez y Castellanos, 2011). Los datos del Gráfico 3 sitúan a España como el cuarto país europeo, con un número de artículos publicados en revistas SCI que representa el $8 \%$ de la producción científica mundial. 
Gráfico 3

Producción científica en economía del deporte por países (2002-2010)

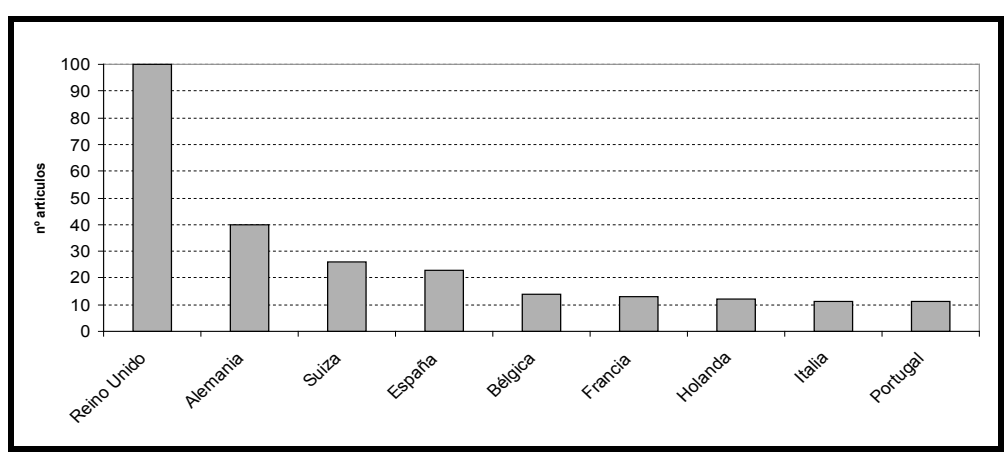

Fuente: Elaboración propia.

Tabla 1

Producción científica en economía del deporte indexada en SCI por países (1990-2009)

\begin{tabular}{|c|c|c|c|c|c|c|}
\hline País & Artículos & (\%) & País & Indice Población* & País & Indice PIB ${ }^{* *}$ \\
\hline USA & 582 & 54.19 & Suiza & 2.79 & N. Zelanda & 0.0615 \\
\hline Reino Unido & 146 & 13.59 & Reino Unido & 2.37 & Reino Unido & 0.0546 \\
\hline Canada & 76 & 7.08 & Canada & 2.29 & Canada & 0.0506 \\
\hline Alemania & 40 & 3.72 & N. Zelanda & 1.87 & Suiza & 0.0427 \\
\hline Australia & 28 & 2.61 & USA & 1.87 & USA & 0.0413 \\
\hline Suiza & 21 & 1.96 & Noruega & 1.47 & Israel & 0.0396 \\
\hline España & 19 & 1.77 & Bélgica & 1.42 & Portugal & 0.0370 \\
\hline Bélgica & 15 & 1.40 & Australia & 1.33 & Bélgica & 0.0298 \\
\hline Holanda & 14 & 1.30 & Israel & 1.13 & Australia & 0.0276 \\
\hline Francia & 12 & 1.12 & Holanda & 0.85 & Chile & 0.0237 \\
\hline Japón & 12 & 1.12 & Portugal & 0.84 & Holanda & 0.0161 \\
\hline Corea del Sur & 12 & 1.12 & Dinamarca & 0.55 & Noruega & 0.0155 \\
\hline China & 11 & 1.02 & Alemania & 0.49 & Suráfrica & 0.0145 \\
\hline Italia & 9 & 0.84 & Grecia & 0.45 & Grecia & 0.0140 \\
\hline Portugal & 9 & 0.84 & España & 0.43 & Rep. Checa & 0.0140 \\
\hline Israel & 8 & 0.74 & Rep. Checa & 0.29 & Corea del Sur & 0.0129 \\
\hline N. Zelanda & 8 & 0.74 & Corea del Sur & 0.25 & España & 0.0118 \\
\hline Noruega & 7 & 0.65 & Chile & 0.24 & Alemania & 0.0110 \\
\hline Grecia & 5 & 0.47 & Francia & 0.19 & Dinamarca & 0.0088 \\
\hline Chile & 4 & 0.37 & Italia & 0.15 & Taiwan & 0.0074 \\
\hline Suráfrica & 4 & 0.37 & Taiwan & 0.13 & Francia & 0.0042 \\
\hline Rep. Checa & 3 & 0.28 & Japón & 0.09 & Italia & 0.0039 \\
\hline Dinamarca & 3 & 0.28 & Suráfrica & 0.08 & China & 0.0025 \\
\hline Taiwan & 3 & 0.28 & China & 0.01 & Japón & 0.0024 \\
\hline
\end{tabular}

* Número de publicaciones por millón de habitantes.

** Número de publicaciones por millardo de dólares USA de PIB.

Fuente: Sánchez y Castellanos (2011). 
En aras de una contextualización más precisa de la posición relativa que ocupa España en el concierto internacional, en la Tabla 1 puede verse una comparación entre países de la producción científica en SCI teniendo en cuenta la población y el PIB. En ambos casos, España desciende posiciones con respecto al ranking construido sin corregir por ambas variables. No obstante, cualquier valoración del puesto que ocupa España no debería ser ajena al hecho de que dichos datos corresponden al período 1990-2010 cuando, como hemos mencionado anteriormente, el primer registro de nuestra base de datos es de 2002. De este modo, que el posicionamiento a nivel internacional que reflejan los datos no sea más favorable se debe en gran parte al hecho de que el interés por la investigación en economía del deporte es relativamente más reciente en nuestro país.

\subsection{Revistas en las que se publica la investigación en economía del deporte}

Otra de las vertientes a tener en cuenta en el marco de este análisis bibliométrico es concerniente a las revistas en las que publican los investigadores españoles en economía del deporte. Los 70 artículos incluidos en nuestra base de datos se publicaron en 42 revistas, dato este que revela que el abanico de revistas con trabajos sobre economía del deporte es bastante amplio, dado que, del total de revistas, únicamente 9 se pueden considerar como especializadas en el ámbito del deporte. El Gráfico 4 muestra aquellas revistas que contienen al menos dos artículos. Tal y como puede comprobarse en el mencionado gráfico, sólo en once revistas $(26,2 \%)$ se publicó más de un artículo.

Gráfico 4

Revistas más frecuentes

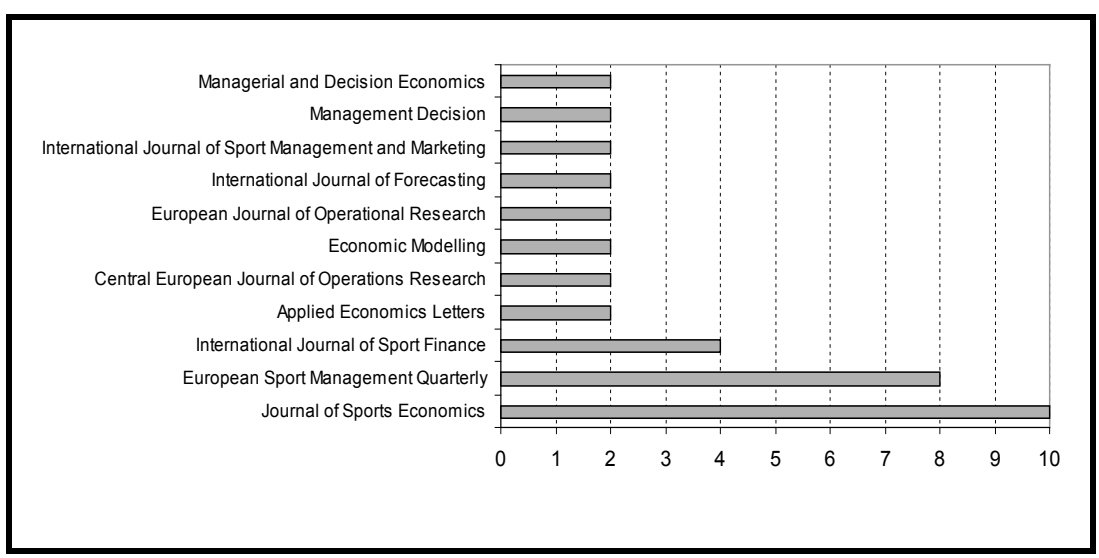

Fuente: Elaboración propia. 
En términos generales, cabe subrayar que el $45 \%$ de los trabajos se publican en revistas relacionadas con el mundo del deporte; de hecho tres revistas acaparan el 30\% del total de publicaciones contempladas en este estudio. No obstante, resulta destacable que la mayoría de la producción se ha publicado en revistas más generalistas o de otros campos de especialización, lo cual sugiere que el interés de los resultados que se derivan de las investigaciones no se circunscribe únicamente al ámbito del deporte. Prueba de ello es que algunos de los artículos se publican en revistas de elevado impacto en el ámbito de la Economía (American Economic Review, Review of Economics and Statistics, etc.)

Por otra parte, en lo que se refiere a la calidad de la investigación en economía del deporte realizada por investigadores españoles, en la medida en que ésta se pueda aproximar por la calidad de la revista en la que se publica, el rasgo más destacable es que el $75,3 \%$ de los trabajos están publicados en revistas JCR con índice de impacto, elemento este que cabe interpretar como indicador positivo de la calidad media de la investigación que se produce en este campo.

Además, en esta misma línea también constituye un dato interesante que el factor de impacto medio de las revistas en las que los investigadores españoles en economía del deporte publican sus artículos es de 0,861 , cifra esta equiparable a la que arrojan los resultados a nivel internacional $(0,90)$. Como elemento de referencia para valorar en su justa medida estos valores, cabe comentar de acuerdo con los datos obtenidos de Thompson ISI (2010), el factor de impacto medio de las revistas del área de Economía es de 1,16.

Finalmente, uno de los aspectos dignos de mención es la escasez de revistas españolas; de hecho, la presencia es testimonial (sólo dos revistas de economía) y ninguno en revistas de impacto. Este dominio absoluto de las publicaciones en revistas internacionales, en cierto modo puede catalogarse como algo insólito, sobre todo si se compara con otras disciplinas, como economía de la cultura, economía de la salud, economía del transporte, etc.

Entre las posibles razones que explican esta realidad, muy probablemente se encuentran las mismas que apunta Herrero (2009) para el caso de la investigación en economía de la cultura. A saber: la inexistencia de una revista nacional especializada, el reducido impacto o reconocimiento que todavía tienen estos temas en el ámbito académico español y la preferencia de los autores españoles por publicar fuera (búsqueda de visibilidad, mayor impacto, incentivos de promoción académica, etc.).

\subsection{Clasificación temática de las publicaciones y metodologías empleadas}

La determinación de las temáticas analizadas en los artículos y las metodologías aplicadas se ha obtenido mediante la aplicación de un análisis exploratorio del contenido de cada uno de los artículos. 
En el ámbito general de la economía del deporte, la diversidad temática es muy amplia. En este sentido, no se dispone de una clasificación establecida que permita codificar los temas abordados en los trabajos, con lo cual las categorías que se delimiten dependerán del criterio utilizado para la clasificación. En nuestro caso, la clasificación temática de los artículos se ha realizado de acuerdo con un criterio basado en las agrupaciones temáticas más frecuentes en los manuales más conocidos de economía del deporte. En líneas generales, y en lo que se refiere a la adscripción temática de los artículos de nuestra muestra, efectuamos una doble clasificación atendiendo a los deportes objeto de estudio (Gráfico 5) y a las cuestiones específicas que se abordan en los trabajos (Gráfico 6).

En el Gráfico 5 queda patente que el deporte profesional de equipo es el que recibe mayor atención y, dentro de éste, el fútbol. No en vano el $60 \%$ de la investigación en economía del deporte gira en torno al fútbol.

\section{Gráfico 5}

Deportes más investigados

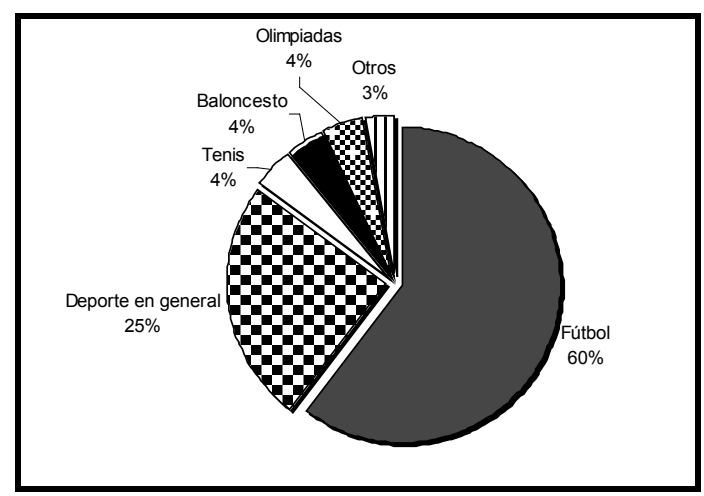

Fuente: Elaboración propia.

Por su parte, en el Gráfico 6 se constata que los temas más frecuentes son, por este orden: eficiencia y productividad (a gran distancia de los demás), demanda, impacto económico y apuestas deportivas. En términos comparativos, se observan algunas diferencias sustanciales con respecto a la distribución temática de la producción científica en esta área a nivel internacional. De hecho, el aspecto más llamativo es la práctica ausencia de artículos que aborden el mercado de trabajo de los deportistas profesionales, siendo éste, a nivel internacional, el tema más tratado dentro de la disciplina. Posiblemente, esta realidad no es ajena a la escasez de datos en España sobre remuneraciones de los deportistas profesionales. 
Gráfico 6

Temática de los artículos

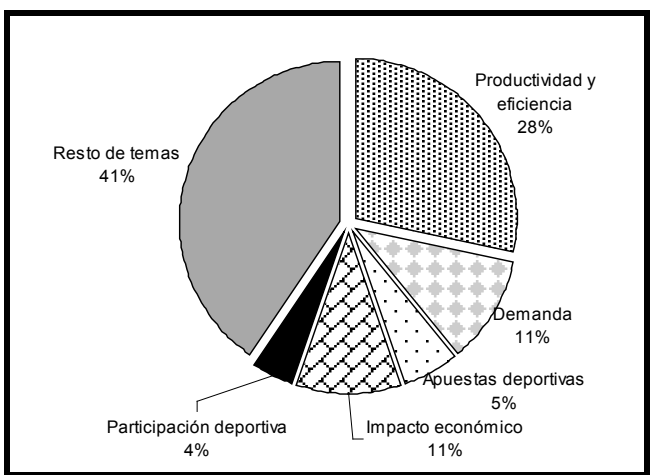

Fuente: Elaboración propia.

En cuanto a las metodologías utilizadas en los artículos publicados sobre economía del deporte, se observa una preferencia por el uso de métodos de investigación empíricos. Las metodologías más usadas son las cuantitativas $\mathrm{y}$, en particular, los análisis de regresión.

\subsection{Producción científica por autores: coautorías, productividad, concentración e impacto}

Una de las cuestiones claves que se suelen evaluar en este tipo de estudios bibliométricos es la relativa a las coautorías. El índice de autoría se determina en función del número de firmantes por artículo y a partir de esto se pueden clasificar en artículos de autoría individual y autoría compartida. La distribución de artículos según el número de autores aparece reflejada en la Tabla 2. De acuerdo con las cifras de esta tabla, se observa una marcada inclinación hacia las autorías colectivas. En concreto, el peso de los artículos de autor único es muy reducido $(5,5 \%)$, mientras que en su mayoría los artículos publicados en las diferentes revistas son firmados por dos o tres autores. Cabe subrayar que algo más de la mitad $(50,6 \%)$ de todas las publicaciones son firmadas por tres investigadores.

A partir de los datos de la muestra, el indicador del grado de colaboración (IC) ${ }^{3}$ se calcula teniendo en cuenta que hay un total de 189 firmas para los 73 trabajos considerados; en este caso, el IC toma el valor de 2,58. Esta cifra es ligeramente mayor que el índice que Bordons y Gómez (1997) establecen para las Ciencias Sociales en España (2 firmas por trabajo). Por otra parte y a efectos comparativos, se podría mencionar que el número medio de autores por artículo

\footnotetext{
${ }^{3}$ El índice de colaboración viene dado por la fórmula: $\mathrm{IC}=\mathrm{n}^{\mathrm{o}}$ firmas $/ \mathrm{n}^{\circ}$ de artículos.
} 
también es superior al de 1,9 que arroja el análisis bibliométrico de la producción científica en economía del deporte a nivel mundial (Sánchez y Castellanos, 2011).

Tabla 2

Importancia de las coautorías

\begin{tabular}{ccccc}
\hline $\mathbf{N}^{\circ}$ AUTORES & $\mathbf{N}^{0}$ ARTICULOS & $\%$ & $\mathbf{N}^{\circ}$ PAGINAS & $\%$ \\
\hline 1 & 4 & 5,5 & 76 & 6,6 \\
2 & 33 & 45,2 & 532 & 46,5 \\
3 & 26 & 35,6 & 358 & 31,3 \\
4 & 9 & 12,3 & 162 & 14,1 \\
5 & 1 & 1,4 & 17 & 1,5 \\
TOTAL & $\mathbf{7 3}$ & $\mathbf{1 0 0 , 0}$ & $\mathbf{1 . 1 4 5}$ & $\mathbf{1 0 0 , 0}$ \\
\hline
\end{tabular}

Fuente: Elaboración propia.

Asimismo, también cabe apuntar la coautoría de investigadores de distintas nacionalidades $\mathrm{y} / \mathrm{o}$ de diferentes universidades, lo cual puede interpretarse como un signo positivo de la colaboración entre autores, el fortalecimiento de grupos de trabajo y el aumento en la comunicación científica, elementos todos indispensables para el desarrollo de la ciencia. A este respecto, si medimos el grado de internacionalización a partir del número de artículos que cuentan con autores de otros países, además de los españoles, constatamos que un $26 \%$ de los artículos se han realizado en colaboración con autores de otros países, especialmente europeos.

Por otro lado, para examinar la productividad de los investigadores españoles en este campo, hemos ponderado el número de artículos y de páginas por la razón del número de participantes en la elaboración de cada artículo. La Tabla 3 recoge el valor absoluto y ponderado de ambas variables (artículos y páginas) para un listado de autores que cuentan al menos con dos publicaciones en economía del deporte.

Uno de los aspectos que tradicionalmente se aborda en los estudios bibliométricos con el fin de evaluar el estado de consolidación de un campo de investigación, es la evolución de la concentración de producción científica por autores. Aunque en este caso, dado el reducido número de años en el que contamos con un número de artículos significativo, no se pueden extraer tendencias temporales, sí se pueden apuntar algunos datos que resultan ilustrativos, especialmente si se comparan con los de la producción científica en economía del deporte a nivel global. 
Tabla 3

Producción científica de investigadores españoles en economía del deporte

\begin{tabular}{|c|c|c|c|c|c|c|c|}
\hline Autor & $\begin{array}{c}\mathrm{N}^{\circ} \\
\text { publicaciones }\end{array}$ & Autor & $\begin{array}{c}\mathrm{N}^{\circ} \\
\text { publicaciones } \\
\text { ponderadas }\end{array}$ & Autor & $\begin{array}{l}\text { Páginas } \\
\text { Totales }\end{array}$ & Autor & $\begin{array}{l}\text { Páginas } \\
\text { ponderadas }\end{array}$ \\
\hline Rodríguez Guerrero, P. & 10 & Rodríguez Guerrero, P. & 4,25 & Rodríguez Guerrero, P. & 157 & Rodríguez Guerrero, P. & 68,67 \\
\hline García Villar, J. & 9 & García Villar, J. & 3,83 & García Villar, J. & 152 & García Villar, J. & 65,83 \\
\hline García del Barrio, P. & 8 & García del Barrio, P. & 3,50 & Castellanos García, P. & 115 & Castellanos García, P. & 62,67 \\
\hline Del Corral Cuervo, J. & 6 & Del Corral Cuervo, J. & 2,83 & García del Barrio, P. & 114 & García del Barrio, P. & 51,17 \\
\hline Castellanos García, P. & 5 & Castellanos García, P. & 2,67 & Lera López, F. & 96 & Del Corral Cuervo, J. & 44,50 \\
\hline Espitia Escuer, M. A. & 5 & Espitia Escuer, M. A. & 2,50 & Sánchez Santos, J. M. & 91 & Lera López, F. & 42,67 \\
\hline Lera López, F. & 5 & Lera López, F. & 2,17 & Del Corral Cuervo, J. & 89 & Espitia Escuer, M. A. & 39,50 \\
\hline García Cebrián, L. I. & 4 & García Cebrián, L. I. & 2,00 & Espitia Escuer, M. A. & 79 & Sánchez Santos, J. M. & 38,67 \\
\hline Liern Carrión, V. & 4 & Sánchez Santos, J. M. & 1,67 & Liern Carrión, V. & 77 & Rapún Gárate, M. & 32,00 \\
\hline Sala Garrido, V. & 4 & Rapún Gárate, M. & 1,50 & Sala Garrido, V. & 77 & García Cebrián, L. I. & 30,50 \\
\hline Sánchez Santos, J. M. & 4 & González Gómez, F. & 1,33 & Rapún Gárate, M. & 64 & García Sánchez, I. M. & 25,00 \\
\hline Boscá Mares, J. E. & 3 & Picazo Tadeo, A. J. & 1,33 & García Cebrián, L. I. & 61 & González Gómez, F. & 23,83 \\
\hline Burillo Naranjo, $P$. & 3 & Prieto Rodríguez, J. & 1,17 & Boscá Mares, J. E. & 51 & Picazo Tadeo, A. J. & 23,83 \\
\hline Gallardo Guerrero, L. & 3 & García Sánchez, I. M. & 1,00 & Martínez Estévez, A. & 51 & Liern Carrión, V. & 19,25 \\
\hline González Gómez, F. & 3 & Liern Carrión, V. & 1,00 & González Gómez, F. & 49 & Sala Garrido, V. & 19,25 \\
\hline Martínez Estévez, A. & 3 & Nicolau Gonzálbez, J. L. & 1,00 & Picazo Tadeo, A. J. & 49 & Pujol Torras, F. & 18,50 \\
\hline Picazo Tadeo, A. J. & 3 & Pujol Torras, F. & 1,00 & Prieto Rodríguez, J. & 44 & Prieto Rodríguez, J. & 16,83 \\
\hline Prieto Rodríguez, J. & 3 & Sala Garrido, V. & 1,00 & Burillo Naranjo, P. & 38 & Suárez Fernández, Ma. J. & 14,33 \\
\hline Rapún Gárate, M. & 3 & Gómez Ruano, M. A. & 0,83 & Gallardo Guerrero, L. & 38 & Burillo Naranjo, P. & 13,40 \\
\hline Barajas Alonso, Á. & 2 & Palacios Huerta, I. & 0,83 & Pujol Torras, F. & 37 & Gallardo Guerrero, L. & 13,40 \\
\hline Campos López, J. C. & 2 & Pérez Carcedo, L. & 0,83 & Barajas Alonso, Á. & 34 & Aizpurua Aguirre, J. & 13,00 \\
\hline García Tascón, M. & 2 & Barajas Alonso, Á. & 0,75 & Campos López, J. C. & 32 & Llorente Erviti, L. & 13,00 \\
\hline Gómez Ruano, M. A. & 2 & Boscá Mares, J. E. & 0,75 & Paramio Salcines, J. L. & 32 & Boscá Mares, J. E. & 12,75 \\
\hline Lozano Segura, S. & 2 & Martínez Estévez, A. & 0,75 & Palacios Huerta, I. & 26 & Martínez Estévez, A. & 12,75 \\
\hline Palacios Huerta, I. & 2 & Campos López, J. C. & 0,67 & García Sánchez, I. M. & 25 & Palacios Huerta, I. & 11,50 \\
\hline Paramio Salcines, J. L. & 2 & Paramio Salcines, J. L. & 0,67 & Lozano Segura, S. & 25 & Campos López, J. C. & 10,67 \\
\hline Pérez Carcedo, L. & 2 & Tena Horrillo, J. D. & 0,67 & Tena Horrillo, J. D. & 25 & Paramio Salcines, J. L. & 10,67 \\
\hline Pujol Torras, F. & 2 & Lozano Segura, S. & 0,58 & Pérez Carcedo, L. & 15 & Tena Horrillo, J. D. & 8,33 \\
\hline Suárez Fernández, Mª.J. & 2 & Burillo Naranjo, P. & 0,45 & Gómez Ruano, M. A. & 13 & Lozano Segura, S. & 7,42 \\
\hline Tena Horrillo, J. D. & 2 & Gallardo Guerrero, L. & 0,45 & Nicolau Gonzálbez, J. L. & 8 & Gómez Ruano, M. A. & 5,33 \\
\hline
\end{tabular}

Fuente: Elaboración propia.

En el caso de la economía del deporte en España, los cinco primeros autores con mayor producción científica acaparan el $27 \%$ del total, los diez primeros el $41 \%$ y los 25 primeros el $63,4 \%$. Estas mismas cifras para la producción científica global en economía del deporte en SCI en el período 2000-2009 son del $17,39 \%$ para los diez primeros y $36,28 \%$ para los veinticinco primeros. Por otra parte si se utiliza un índice de Herfindhal, el valor que se obtiene para el periodo analizado en España (2002-11) es de 262 y en el contexto internacional (media 
del período 2000-2009) de 130. A efectos de visualizar esta realidad, en el Gráfico 7 se representa una curva de Lorenz para la producción científica en economía del deporte en España que refleja claramente que el nivel de concentración es relativamente alto.

\section{Gráfico 7}

Curva de Lorenz de la producción científica de investigadores españoles en economía del deporte

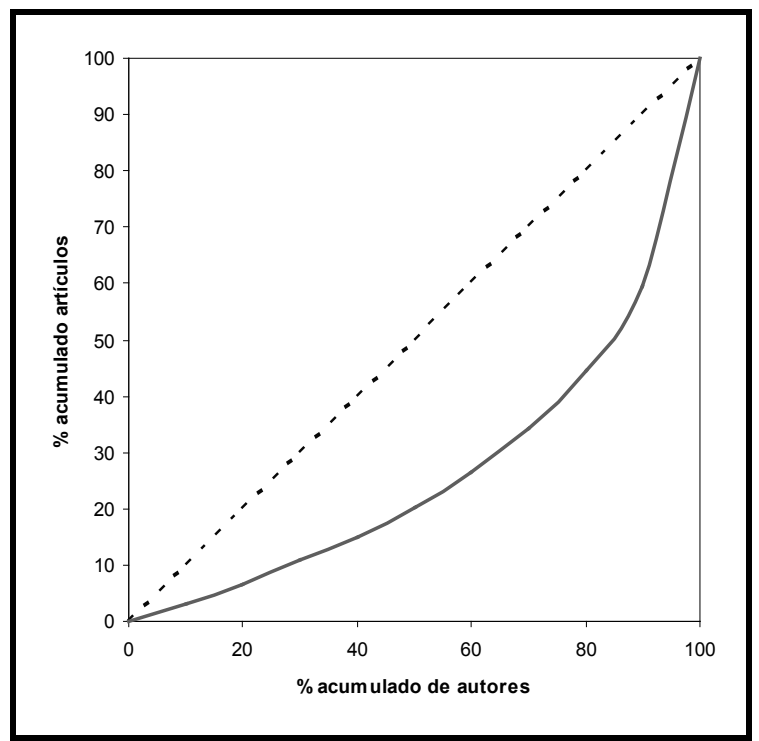

Fuente: Elaboración propia.

Por lo tanto, estos datos sugieren que en España la disciplina no está tan consolidada como a nivel internacional. No obstante, cabría esperar que, en la medida en que continúe aumentando el número de investigadores españoles interesados por la economía del deporte, el grado de concentración antes mencionado tienda a reducirse en el tiempo y la disciplina complete su proceso de consolidación.

Un aspecto relevante de toda producción científica es el impacto que ésta tiene en la comunidad internacional. En el caso de la investigación española en economía del deporte, si bien en términos cuantitativos la producción no es muy alta, sí ha tenido cierta repercusión a nivel de citas. Evidentemente, las posibilidades de realizar un análisis de citas exhaustivo están enormemente limitadas por el reducido intervalo temporal considerado. El estudio de las pautas de evolución de las citas, requeriría analizar las publicaciones de los distintos años 
considerando una ventana de citas de cinco años, por ejemplo, procedimiento este que no cabe aplicar a la muestra de nuestro trabajo.

No obstante, a efectos ilustrativos cabría señalar que para el total de las 73 publicaciones objeto de estudio, se obtienen 120 citas en SSCI (21 artículos citados), 177 citas en Scopus (25 artículos citados) y 453 citas en Google Académico (57 artículos citados). ${ }^{4}$ Como información adicional sobre el impacto de la investigación en economía del deporte realizada por autores españoles -y que, por supuesto, hay que valorar con todas las cautelas-, en las Tablas 4, 5 y 6 , ofrecemos los 10 artículos más citados en dos de las bases de datos utilizadas como referencia y, como complemento, las citas en Google Académico.

\section{Tabla 4}

Artículos más citados en SSCl y en SCl

\begin{tabular}{clcc}
\hline 1 & $\begin{array}{l}\text { Garicano, L. y Palacios, I. (2005): Favoritism under social pressure, Review of } \\
\text { Economics and Statistics }\end{array}$ & 30 \\
\hline 2 & $\begin{array}{l}\text { Lozano, S.; Villa, G.; Guerrero, F. y Cortes, P. (2002): Measuring the performance of } \\
\text { nations at the Summer Olympics using data envelopment analysis, Journal of the } \\
\text { Operational Research }\end{array}$ & 16 \\
\hline 3 & $\begin{array}{l}\text { Lera, F. y Rapún, M. (2007): The demand for sport: Sport consumption and } \\
\text { participation models, Journal of Sport Management }\end{array}$ & 10 \\
\hline 4 & $\begin{array}{l}\text { Martínez Caro, L. y Martínez García, J. A. (2007), Cognitive-affective model of } \\
\text { consumer satisfaction. An explanatory study within the framework of a sporting event, } \\
\text { Journal of Business Research }\end{array}$ & 8 \\
\hline 5 & $\begin{array}{l}\text { Boscá, J. E.; Liern, V.; Martínez Estévez, A. y Sala, R. (2009): Increasing offensive or } \\
\text { defensive efficiency? An analysis of Italian and Spanish football, Omega-International } \\
\text { Journal of Management Science }\end{array}$ & 7 \\
\hline 6 & $\begin{array}{l}\text { Cooper, W.; Ruiz, J. L. y Sirvent, I. (2009): Selecting non-zero weights to evaluate } \\
\text { effectiveness of basketball players with DEA, European Journal of Operational } \\
\text { Research }\end{array}$ & 6 \\
\hline 6 & $\begin{array}{l}\text { García Sánchez, I. M. (2007): Efficiency and effectiveness of Spanish football teams: A } \\
\text { three-stage-DEA approach, Central European Journal of Operations Research }\end{array}$ & 6 \\
\hline 7 & $\begin{array}{l}\text { Barros, C. y García del Barrio, P. (2008): Efficiency measurement of the English } \\
\text { football Premier League with a random frontier model, Economic Modelling }\end{array}$ & 5 \\
\hline 7 & $\begin{array}{l}\text { Boscá, J. E.; Liern, V.; Martínez Estévez, A. y Sala, R. (2008): The Spanish football } \\
\text { crisis, European Sport Management Quarterly }\end{array}$ & 5 \\
\hline 8 & $\begin{array}{l}\text { Barros, C. y García del Barrio, P. (2009): Analysing the technical efficiency of the } \\
\text { Spanish football league First Division with a random frontier model, Applied Economics }\end{array}$ & 4 \\
\hline
\end{tabular}

Fuente: Elaboración propia.

\footnotetext{
${ }^{4}$ Las citas han sido registradas el 15 de marzo de 2012. Obviamente, una fecha diferente de cómputo arrojará cifras diferentes, dado que estas bases de datos se actualizan continuamente.
} 
Tabla 5

Artículos más citados en Scopus

\begin{tabular}{clc}
\hline 1 & $\begin{array}{l}\text { Garicano, L. y Palacios, I. (2005): Favoritism under local pressure, Review of } \\
\text { Economics and Statistics }\end{array}$ & 33 \\
\hline 2 & $\begin{array}{l}\text { Lozano, S.; Villa, G.; Guerrero, F. y Cortes, P. (2002): Measuring the performance of } \\
\text { nations at the Summer Olympics using data envelopment analysis, Journal of the } \\
\text { Operational Research }\end{array}$ & 18 \\
\hline 3 & $\begin{array}{l}\text { Martínez Caro, L y Martínez García, J. A. (2007): Cognitive-affective model of } \\
\text { consumer satisfaction. An explanatory study within the framework of a sporting event, } \\
\text { Journal of Business Research }\end{array}$ & 17 \\
\hline 4 & $\begin{array}{l}\text { Espitia, M. y García Cebrián, I. (2006): Performance in sports teams results and } \\
\text { potential in the professional soccer league in Spain, Management Decision }\end{array}$ & 13 \\
\hline 5 & $\begin{array}{l}\text { Cooper, W.; Ruiz, J. L. y Sirvent, I. (2009): Selecting non-zero weights to evaluate } \\
\text { effectiveness of basketball players with DEA, European Journal of Operational }\end{array}$ & 12 \\
\hline 5 & $\begin{array}{l}\text { Lesearch } \\
\text { participation models, Journal of Sport Management }\end{array}$ & 12 \\
\hline 6 & $\begin{array}{l}\text { Boscá, J. E.; Liern, V.; Martínez Estévez, A. y Sala, R. (2009): Increasing offensive or } \\
\text { defensive efficiency? An analysis of Italian and Spanish football, Omega-International } \\
\text { Journal of Management Science }\end{array}$ & 11 \\
\hline 7 & $\begin{array}{l}\text { Barros, C. y Del Corral, J. (2008): Identification of segments of soccer clubs in the } \\
\text { Spanish league First Division with a latent class model, Journal of Sports Economics }\end{array}$ & 9 \\
\hline 7 & $\begin{array}{l}\text { García Sánchez, I. M. (2007): Efficiency and effectiveness of Spanish football teams: A } \\
\text { three-stage-DEA approach, Central European Journal of Operations Research }\end{array}$ & 9 \\
\hline 8 & $\begin{array}{l}\text { Barros, C. y García del Barrio, P. (2008): Efficiency measurement of the English } \\
\text { football Premier League with a random frontier model, Economic Modelling }\end{array}$ & 7 \\
\hline
\end{tabular}

Fuente: Elaboración propia.

Tabla 6

Artículos más citados en Google Académico

\begin{tabular}{clc}
\hline 1 & $\begin{array}{l}\text { Garicano, L. y Palacios, I. (2005): Favoritism under social pressure, Review of } \\
\text { Economics and Statistics }\end{array}$ & 108 \\
\hline 2 & $\begin{array}{l}\text { García Villar, J. y Rodríguez Guerrero, P. (2002): The determinants of football match } \\
\text { attendance revisited: Empirical evidence from the Spanish football league, Journal of } \\
\text { Sports Economics }\end{array}$ & 106 \\
\hline 3 & $\begin{array}{l}\text { Lozano, S.; Villa, G.; Guerrero, F. y Cortes, P. (2002): Measuring the performance of } \\
\text { nations at the Summer Olympics using data envelopment analysis, Journal of the } \\
\text { Operational Research }\end{array}$ & 51 \\
\hline 4 & $\begin{array}{l}\text { Martínez Caro, L y Martínez García, J. A. (2007): Cognitive-affective model of } \\
\text { consumer satisfaction. An explanatory study within the framework of a sporting event, }\end{array}$ & 46 \\
\hline Journal of Business Research & 34 \\
\hline 5 & $\begin{array}{l}\text { Espitia, M. (2004): Measuring the efficiency of Spanish First-Division soccer teams, } \\
\text { Journal of Sports Economics }\end{array}$ & 27 \\
\hline 6 & $\begin{array}{l}\text { Cooper, W.; Ruiz, J. L. y Sirvent, I. (2009): Selecting non-zero weights to evaluate } \\
\text { effectiveness of basketball players with DEA, European Journal of Operational }\end{array}$ & 32 \\
\hline Research & $\begin{array}{l}\text { Espitia, M. y García Cebrián, I. (2006): Performance in sports teams results and } \\
\text { potential in the professional soccer league in Spain, Management Decision }\end{array}$ & 20 \\
\hline 8 & $\begin{array}{l}\text { Boscá, J. E.; Liern, V.; Martínez Estévez, A. y Sala, R. (2009): Increasing offensive or } \\
\text { defensive efficciency? An analysis of Italian and Spanish football, Omega-International }\end{array}$ & 20 \\
\hline Journal of Management Science \\
\hline $\begin{array}{l}\text { García del Barrio, P. y Pujol, F. (2007): Hidden monopsony rents in winner-take-all } \\
\text { markets - Sport and economic contribution of Spanish soccer players, Managerial and } \\
\text { Decision Economics }\end{array}$ & 19 \\
\hline 9 & $\begin{array}{l}\text { Garcia del Barrio, P. y Szymanski, S. (2009): Goal! Profit maximization versus win } \\
\text { maximization in soccer, Review of Industrial Organization }\end{array}$ & 19 \\
\hline
\end{tabular}

Fuente: Elaboración propia. 


\subsection{Producción científica por universidades}

El Gráfico 8 ilustra aquellas universidades con mayores resultados en términos de número de publicaciones en economía del deporte. Para ello se han seleccionado las instituciones con al menos tres artículos en el decenio 20022011. Tal y como se desprende de la información contenida en el gráfico, tres universidades (Oviedo, Pompeu Fabra y Valencia) concentran más de la mitad de la investigación (53\%), siendo la Universidad de Oviedo la que ocupa el lugar más destacado, tanto en número de trabajos como de investigadores que participan en los mismos, constituyéndose sin duda en un referente institucional en el ámbito de la economía del deporte.

\section{Gráfico 8}

Universidades españolas con mayor producción científica en economía del deporte

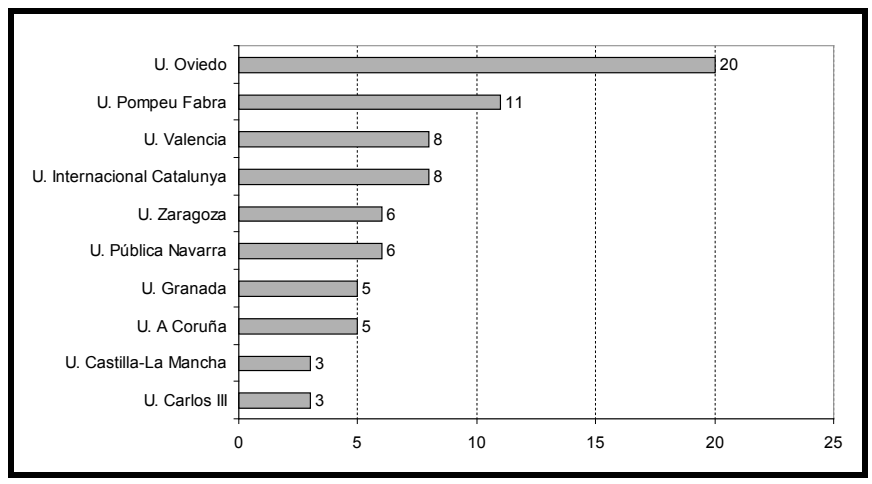

Fuente: Elaboración propia.

\section{CONSIDERACIONES FINALES}

En definitiva y a modo de consideraciones finales, podemos decir que la investigación en economía del deporte en España tiene una historia breve pero prometedora. En concreto, los resultados de este análisis bibliométrico nos permiten constatar los siguientes extremos:

- La economía del deporte es una disciplina emergente, cuando menos incipiente, que en el caso de España, podría afirmarse que está iniciando su proceso de consolidación.

- La investigación en economía del deporte desarrollada por investigadores españoles está adquiriendo una presencia significativa en el concierto internacional.

- La mayoría de la investigación se publica en revistas internacionales de impacto, observándose una práctica ausencia de publicaciones en revistas españolas. 
- En cuanto a los indicadores de calidad que se desprenden de las citas y el impacto de las revistas en que se publica la investigación, los resultados ponen de manifiesto que la calidad media es aceptable y mejora gradualmente.

- El papel de las autorías colectivas, medida en términos de autores por artículo, es relativamente elevado tanto si se compara con la media de las Ciencias Sociales como con la media de la investigación en economía del deporte a nivel internacional

- A pesar de que la muestra es reducida, el análisis nos ha permitido identificar los autores pioneros y los que se mantienen más activos en este campo de estudio. En este sentido, es destacable el elevado grado de concentración de las publicaciones en un reducido número tanto de autores como de universidades.

- La mayoría de las investigaciones se centran en el estudio de los deportes profesionales de equipo y, más en concreto, en el fútbol. De aquí se desprende que hay otras áreas temáticas con un gran potencial para el desarrollo de investigaciones que puedan resultar fructíferas en términos tanto de originalidad como de relevancia de las aportaciones. A título de ejemplo, se puede mencionar el análisis del deporte entendido como práctica o participación deportiva.

- Dado el limitado grado de internacionalización, medida como coautoría con investigadores extranjeros, una de las implicaciones que se deriva de nuestros resultados es la conveniencia de potenciar la misma, ya que cabe esperar que, de acuerdo con lo que sucede en otras disciplinas, las publicaciones en colaboración internacional tengan un impacto más alto que las que cuentan únicamente con autores españoles.

Para dar por concluido este apartado de consideraciones finales, cabe mencionar que algunas de las limitaciones de las que adolece el estudio desarrollado están asociadas al hecho de que la muestra que ha servido de referencia para nuestro análisis es demasiado pequeña para poder aplicar todas las técnicas de un análisis bibliométrico más completo, dado que muchos de los procedimientos están diseñados para obtener resultados significativos con mayores tamaños muestrales.

Por último, en cuanto a posibles extensiones de una investigación de estas características, quizás uno de los aspectos más relevantes a abordar sería el relativo a la evaluación de la influencia de la investigación en un campo específico como es la economía del deporte sobre otras disciplinas. Para ello cabría acometer un análisis más exhaustivo de las pautas de citado; en concreto, cabría analizar el perfil de las publicaciones en las que se citan los artículos de economía del deporte. 


\section{REFERENCIAS BIBLIOGRÁFICAS}

BERRI, D. (2007): Defending sports economics. Disponible en: http://dberri.wordpress.com [1 de diciembre de 2007]

BERRI, D. J. y SCHMIDT, M. B. (2010): Stumbling on wins, New Jersey: FT Press.

BORDONS, M. y GÓMEZ, I. (1997): "La actividad científica española a través de indicadores bibliométricos en el período 1990-1993" en Revista General de Información y Documentación, 7 (2): pp. 69-86.

COATES, D. (2008): What is a sport? What is sports economics? Disponible en: http://thesportseconomist.com [6 de julio de 2008]

FIZEL, J. (2006): "Handbook of sports economics research: an overview". En FIZEL (ed.) Handbook of sports economics research, pp. 3-8, New York and London: M.E. Sharpe.

FORT, R. (2008). What is a sport?/What is sports economics? Disponible en: http://thesportseconomist.com [6 de julio de 2008]

GARCÍA, J. y RODRÍGUEZ, P. (2002): "The determinants of football match attendance revisited: Empirical evidence from the Spanish football league" en Journal of Sports Economics, 3 (1): pp. 18-38.

GOFF, B. y TOLLISON, R. (1990): "Sports as Economics". En GOFF, B. y TOLLISON, R. (eds.): Sportometrics, College Station: Texas A\&M University Press

HERRERO, L. C. (2009): "La investigación en economía de la cultura en España: un estudio bibliométrico" en Estudios de Economía Aplicada, 27 (1): pp. 361388.

JEWELL, R. T. (2006): "Sports economics: the state of the discipline". En Fizel, J., op. cit. (pp. 9-20).

ROTTENBERG, S. (1956): "The baseball players' labor market" en Journal of Political Economy, 64 (3): pp. 242-258.

SÁNCHEZ, J.M. y CASTELLANOS, P. (2011): "A bibliometric análisis of sports economics research" en International Journal of Sport Finance, 6 (3): pp. 222244

THOMPSON ISI (2010). 2009 Journal of Citatitons Reports. Social Science Edition Philadelphia: Thompson ISI.

ZIMBALIST, A. (Ed.) (2001): The economics of sport, Cheltenham: Edward Elgar. 
